\title{
Editorial: Multimedia in Technology Enhanced Learning
}

\author{
Zhigao Zheng $^{1} \cdot$ Jinming Wen ${ }^{2} \cdot$ Shuai Liu ${ }^{3}$
}

Published online: 21 December 2016

(C) Springer Science+Business Media New York 2016

The advent of new technologies such as the Multimedia and Internet of Things has brought opportunities for Technology Enhanced Learning. The technologies with Multimedia can be applied the intelligence functions to learning which range from Smart School, Smart Class to Smart Learning at Home and so on. Accordingly, the assessment and evaluation methods need to be developed and involved into the iterative designing process. This new notion involves some tasks in which traditions are replaced by multimedia technology requiring manual

The original version of this article was revised: The seventh and last paragraphs stating: "In the sixth paper "A Novel Hybrid Approach Based On Chaotic Cloud Gravitational Search Algorithm to Complicated Image Template Matching",...matching problems than the other intelligent optimization algorithms." and "The last article "A Family of High-Order Newton's Iterative Methods in Image Enhancement" proposed a family of third-order...and indicate higher computational efficiency.", were removed.

\section{Zhigao Zheng}

zhengzhigao@pku.edu.cn

Jinming Wen

jinming.wen@ens-lyon.fr

Shuai Liu

cs_liushuai@imu.edu.cn

1 Central China Normal University, Wuhan, China

2 Centre national de la recherché scientifique, Paris, France

3 Inner Mongolia University, Hohhot, China discrimination and resolution to reach the optimization. In addition, with the development of Internet of Things, Technology Enhanced Learning application pays greater attention to the interconnection between multi-modal interactive technologies and information systems to the maximum extent possible. Based these new technologies, the increasing networking in different scales from global to local is having a profound effect on learning and teaching. It makes new forms of collaborative and personalized learning experiences reality. Then it can be seen that further efficient management and deeper analysis of Technology Enhanced Learning are the key tasks in developing smart school or individual learning application at home based on Multimedia technologies.

This special issue features eight selected papers with high quality. The first article, "Greedy Block Coordinate Descent under Restricted Isometry Property", authored by Fumin Zhu investigate the restricted isometry property (RIP) based condition that guarantees exact support recovery of K-group sparse matrices $\mathrm{X}$ from the MMV model with greedy block coordinate descent (GBCD) algorithm in $\mathrm{K}$ iterations. We show that if A satisfies the RIP with $\delta_{K+1}<1 / \sqrt{K+1}$, then GBCD recovers the support of any K-group sparse matrix $X$ in $\mathrm{K}$ iterations. This RIP condition is sharp since GBCD may fail to recover the support of a $\mathrm{K}$-group sparse matrix in $\mathrm{K}$ iterations if $\delta_{K+1} \geq 1 / \sqrt{K+1}$. As far as we know, this is the firstsharp condition for GBCD.

The second article titled "Oblique Projection Matching Pursuit" proposed oblique projection matching pursuit (ObMP) algorithm, which updates the residual in a oblique projection manor. By using the restricted isometric property (RIP), the author builds a solid yet very intuitive grasp of the more accurate phenomenon of ObMP. Empirical experiments 
that the ObMP achieves improved reconstruction performance over the conventional OMP algorithm in terms of support detection ratio and mean squared error (MSE).

In the next article with the title "QR Code Authentication with Embedded Message Authentication Code", this paper proposes to embed the authentication data such as message authentication code and cryptographic signature for QR code authentication. The scheme utilizes the redundancy of the error correction codewords to store the authentication data while still maintaining the default error correction capability. The stego barcode can be decoded by common users with a standard barcode decoder. The embedded data can be extracted with the knowledge of the barcode structure and the embedding process. The authenticity of the QR codes can then be verified off-line by the authorized user as and when needed. Experimental shows that, $100 \%$ of the embedded data and encoded information can be retrieved correctly in the decodable barcodes with the off-the-shelf cameras. Security analysis shows that the proposed scheme is secure against attacks in tampering the barcode content. Compared with the existing data hiding scheme, the proposed method has much higher embedding capacity under the same error correction level.

Current existing banknotes classification methods suffer from time consuming and low accuracy. The fourth paper "A Novel Classification Algorithm for New and Used Banknotes" analyzed the feature of defects and contaminations in a gray-scale image, and then proposed an algorithm to classify new and old banknotes into five levels based on the statistical parameters of the feature points in a gray-scale image, gray level-gradient co-occurrence matrices and a multiDAG-SVM classifier, which can be easily adopted by banknotes sorting applications. Experimental results show that the proposed method is a promising potential application with superior calculating speed and classification efficiency.

Reliable load frequency control (LFC) is crucial to the operation and design of large-scale power system. However, the huge system is always subject to the running uncertainties and external disturbances. Considering the LFC problem in an interconnected power system with four areas, the fifth paper "Robust Distributed Model Predictive Load Frequency Control for Interconnected Power System with State-error" presents a robust distributed model predictive control (RDMPC) based on linear matrix inequalities (LMIs). The proposed algorithm works out problems of local convex constrained optimizations on LFC by minimizing an attractive range for robustness using a time-varying state-feedback controller in each control area. The scheme incorporates the two critical nonlinear constraints, the generation rate constraint (GRC) and the valve limitation, into convex constrained optimization using LMIs even if the state information of the power system may suffer unexpected lose because of the sensor's fault. Furthermore, the algorithm explores the use of an expanded group of adjustable parameters in LMIs to transform an upper bound into an attractive range for shunning conservativeness. Good performance and robustness on load frequency uncertainties and its error state are obtained in the interconnected power system.

With the development of social information technology and the increasing of information data in big data era, how to query the required data accurately is becoming more and more important, paper "Research and Citation Analysis of Data Mining Technology Based on Bayes Algorithm" established a model of data mining technology. In this paper, the author uses the Bayesian network learning model to study the data mining technology. A Bayesian network learning model is established, then, the parameters of the recognition and the selection of coefficients are analyzed in detail, after that, the data mining model based on Bayesian computation is deduced, and the reliability of the model is verified by the example of the students. The probability distribution pattern used by Bayes has many advantages in data mining. It further proves the applicability of Bayesian formula, and provides a reference for data mining technology.

The Markova prediction and analysis is a method based on Markov Chain theory, according to the present states and tendency of some variables, the possible states in a given time in the future will be forecasted. "Stochastic Time Series Analysis for Energy System Based on Markov Chain Model" applies stochastic time series based on Markov Chain Model to predict the developing trend of China's energy consumption structure under the influence of energy policy. This model is adopted to forecast the total amount of energy consumption and the possible trend of energy production and consumption structures. Experimental results demonstrate that this model can effectively simulate and forecast the total amount and structures of primary energy consumption. 
Acknowledgements The guest editors are thankful to our reviewers for their effort in reviewing the manuscripts. We also thank the Edit-in-Chief, Dr. Imrich Chlamtac for his supportive guidance during the entire process. The special issue is supported by National Natural Science Foundation of China Under Grants No. 61502254, No.71471119 and No.71601125, the Development Program for Distinguished Young Teachers in Higher Education of Guangdong Province (Yq2013147).

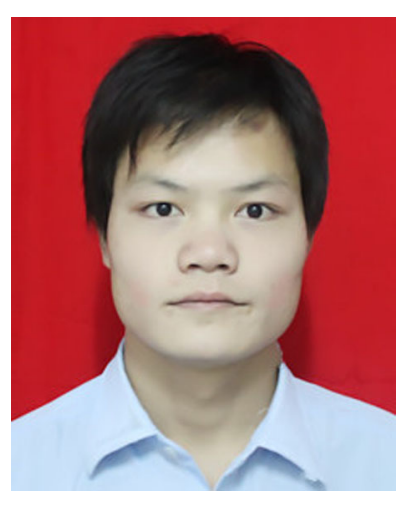

Zhigao Zheng (M12-M12-M16) was an Associate Researcher with the National Engineering Research Centre for E-learning and Collaborative \& Innovative Centre for Educational Technology at Central China Normal University. He is the guest editor of ACM/Springer Mobile Networks and Applications, Multimedia Tools and Applications, Journal of Intelligent \& Fuzzy Systems, Computers \& Electrical Engineering, International Journal of Networking and Virtual Organisations (IJNVO) and so on, he is also the reviewer of many journals such as IEEE Transactions on Big Data, IEEE Transactions on Industrial Informatics, Journal of Network and Computer Applications, The Journal of Supercomputing, Multimedia Tools and Applications and some top conference such as SC'16, CCGrid'16, NPC'15 and NPC'16. His research interests include distributed data stream analysis, cloud computing and graph computing. He became a Member (M) of CCF in 2012, Member (M) of ACM in 2012 and Member (M) of IEEE in 2016.

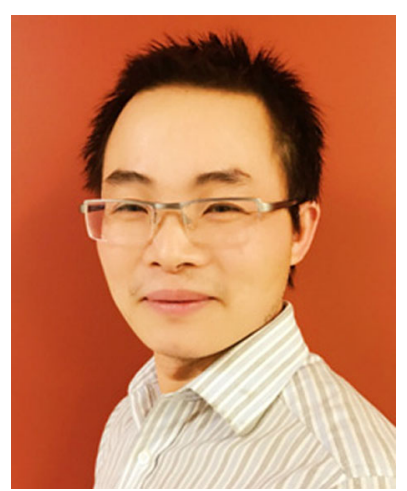

Jinming Wen received his Bachelor degree in Information and Computing Science from Jilin Institute of Chemical Technology, Jilin, China, in 2008, his M. Sc. degree in Pure Mathematics from the Mathematics Institute of Jilin University, Jilin, China, in 2010, and his $\mathrm{Ph} . \mathrm{D}$ degree in Applied Mathematics from McGill University, Montreal, Canada, in 2015. He was a postdoctoral research fellow at Laboratoire LIP, ENS de Lyon from March 2015 to August 2016. He is currently working as a postdoctoral research fellow at department of Electrical and Computer Engineering, University of Alberta. His research interests are in the areas of lattice reduction with applications in communications, signal processing and cryptography, and sparse recovery. He was a Guest Editor for 4 special issues including one in ACM/Springer Mobile Networks and Applications.

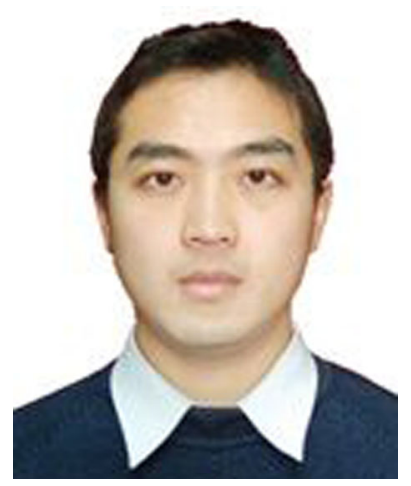

Shuai Liu is current an associate professor at Inner Mongolia University, China. He serves or is serving as an editor or guest editor for many technical journals. He published more than 20 papers in Elsevier and Springer. His interesting research domains contain fractal application and image processing, computer vision. 